\title{
Downregulation of miR-146a promotes proliferation and migration of AOB-treated embryoid body via PDGFRA induction
}

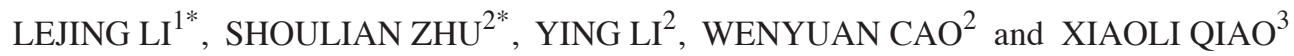 \\ Departments of ${ }^{1}$ Acupuncture and Moxibustio, ${ }^{2}$ Neurology and ${ }^{3}$ Traditional Chinese Medicine, \\ Linzi District People's Hospital, Linzi, Shandong 255400, P.R. China
}

Received March 27, 2017; Accepted August 24, 2017

DOI: $10.3892 / \mathrm{mmr} .2017 .8133$

\begin{abstract}
Antioxidant of bamboo leaves (AOB) has been proven to have antioxidant activity and an inhibitory effect on free radicals that induce deterioration of macromolecules. The multi-target regulation of microRNAs (miRs) in the complicated process of vasculogenesis and angiogenesis lead to the use of miRNA therapy in vascular development. In the present study, the role of miRNAs on early embryo vascular development upon AOB stimulation was investigated. For this purpose, mouse embryonic stem cells were spontaneously differentiated as embryoid bodies (EBs) and were examined by phase contrast microscopy. miR-146a mimic and scramble control were transfected into EBs and potential targets of miR-146a were predicted. Cell proliferation and migration were detected by cell viability and wound-healing and migration assays, respectively. Angiogenesis was determined by the Spheroid sprouting assay. It was demonstrated that EBs transfected with miR-146a mimic had an increased growth rate compared with the control cells. miR-146a-transfected cells were very susceptible to AOB treatment. Furthermore, among the predicted miR-146a targets, platelet-derived growth factor receptor alpha (PDGFRA) was identified as a bona fide target of miR-146a. In conclusion, PDGFRA was demonstrated to participate in the modulation of cell migration and proliferation of mouse EBs. The present study expanded the current understanding of AOB biology and elucidated the mechanisms underlying early embryo vascular development upon AOB stimulation.
\end{abstract}

Correspondence to: Dr Xiaoli Qiao, Department of Traditional Chinese Medicine, Linzi District People's Hospital, 146 Huangong Road, Linzi, Shandong 255400, P.R. China

E-mail:mm11baobei@163.com

Dr Wenyuan Cao, Department of Neurology, Linzi District People's Hospital, 146 Huangong Road, Linzi, Shandong 255400, P.R. China E-mail: girlsflower@163.com

${ }^{*}$ Contributed equally

Key words: embryoid body, angiogenesis antioxidant of bamboo leaves, micro RNA-146a, platelet-derived growth factor receptor alpha, vascular endothelial growth factor

\section{Introduction}

Antioxidant of bamboo leaves (AOB) consists mainly of several bioactive flavonoids (orientin, isoorientin, vitexin and isovitexin), and has been approved by the Ministry of Health of the People's Republic of China as a natural food additive since $2007(1,2)$. $\mathrm{AOB}$ has anti-cancer and cardiovascular protective functions; however, its effects on early embryo vascular development is not well known (3). Platelet-derived growth factor receptor alpha (PDGFRA) is identified as a functional gene that is restrictedly expressed during in vitro differentiation and mouse embryogenesis (4). PDGFRA expression is evident in undifferentiated mouse embryonic stem cells (ESCs), at sites of blood island formation and vasculogenesis during early embryogenesis, as well as during angiogenesis in the adult mice (5). MicroRNAs (miRNAs/miRs) are non-coding single-stranded RNAs that can bind to the 3'-untranslated regions of target mRNAs, leading to their translation or degradation $(6,7)$. miRNAs participate in various biological processes (8) and it has been highlighted that miRNAs serve an important role in early embryo vascular development through promoting or inhibiting angiogenesis (9).

To address whether miRNAs have an important role in early embryo vascular development, the miRNA profile in embryonic bodies (EBs) in response to AOB treatment was investigated. In the current study, subtle alterations in the miRNA profile in EBs following AOB treatment were identified. Through functional enrichment analysis and target prediction, few candidate pathways as putative targets of miR-146a were identified. In the 'Wnt signaling pathway' which was among the top ranked predictions, PDGFRA was verified as a functional target in angiogenesis. Additionally, knockdown of PDGFRA could significantly inhibit the proliferation and migration of EBs.

\section{Materials and methods}

ESC culture. Wild type mouse ESCs (WT ESCs; strain 129; ESD3 cells from the American Type Culture Collection, Manassas, VA, USA) were plated in a flask at a density of 35,000 cells $/ \mathrm{cm}^{2}$ and incubated at $37^{\circ} \mathrm{C}$ with $5 \% \mathrm{CO}_{2}$ for $24 \mathrm{~h}$. ESCs were firstly expanded on a mitotically inactivated feeder layer followed by at least one passage on gelatin-coated plastic in Dulbecco's modified Eagle's medium (DMEM) containing $15 \%$ ESC-qualified fetal bovine serum (FBS; Invitrogen; Thermo Fisher Scientific, Inc., Waltham, MA, USA), 2 mM 
L-glutamine, $0.1 \mathrm{mM}$ non-essential amino acids, $0.1 \mathrm{mM}$ beta-mercaptoethanol, 1,000 U/ml Leukemia Inhibitory Factor (LIF; EMD Millipore, Billerica, MA, USA), 100 U/ml penicillin and $100 \mu \mathrm{g} / \mathrm{ml}$ streptomycin (Sigma-Aldrich; Merck KGaA, Darmstadt, Germany). The study was approved by the Ethics Committee of Linzi District People's Hospital (Linzi, China).

$E B$ differentiation. WT ESCs were spontaneously differentiated as EBs in 3-D suspension culture. ESCs were dissociated and cultured at a density of 800 cells/well for $24 \mathrm{~h}$ at $37^{\circ} \mathrm{C}$. Subsequently, EBs were transferred to culture dishes (Bio-Rad Laboratories, Inc., Hercules, CA, USA) with coated agar and without the feeder layer and LIF, and cultured for up to 10 days. From day 4 onwards, medium and dishes were changed every $24 \mathrm{~h}$.

Morphological assessment. The morphology of pluripotent cell colonies and EBs were determined by using phase contrast microscopy. The size of EBs was evaluated using Image J software (version 3.4; National Institutes of Health, Bethesda, MD, USA) after taking cross sectional areas and phase contrast images for EBs.

Transfection of miR-146a antagomir and mimic, and infection of lentiviral PDGFRA small interfering (si)RNA. miR-146a mimic (cat. no. 4464066; 5'-UGAGAACUGAAUUCC AUGGGUU-3'), antagomir (cat. no. 4464084; 5'-AACCCA UGGAAUUCAGUUCUCA-3') and scramble control (cat. no. 4464058) were purchased from Ambion; Thermo Fisher Scientific, Inc. $10 \mathrm{ng}$ miRNA mimic, antagomir and scramble control was transfected into EBs using Lipofectamine ${ }^{\circledR}$ RNAi Max (Thermo Fisher Scientific, Inc.) for $6 \mathrm{~h}$ at room temperature. Subsequently, cells were harvested and stored at $4^{\circ} \mathrm{C}$ for further assays. Pooled lentiviral siRNA targeting PDGFRA (cat. no. sc-29444-V) and scramble siRNA as a negative control (cat. no. sc-108080) were purchased from Santa Cruz Biotechnology, Inc. (Dallas, TX, USA). Infection with the lentivirus expressing PDGFRA or scramble siRNA was performed as described previously (10).

miRNA microarray analysis. RNA quantity and quality was assessed by using an Agilent 2100 Bioanalyzer. The complementary (c)RNA was generated from $100 \mathrm{ng}$ of total RNA using the FlashTag ${ }^{\mathrm{TM}}$ HSR labeling kit (Genisphere LLC, Hatfield, PA, USA) following the manufacturer's protocol. The cRNA was biotinylated to hybridize the GeneChip miRNA Array (Affymetrix; Thermo Fisher Scientific, Inc.). An Affymetrix Model 3000 scanner was used for scanning arrays. miRNA QC tool software (version 5.1; Affymetrix; Thermo Fisher Scientific, Inc.) was used to generate expression values, which were normalized and log transformed. Differential expression, defined as $\log 2$ fold change $\geq 1.5$ or $\leq-1.5(\mathrm{P}<0.05)$, was detected by the limma tool, in R (version 3.4.2; www.r-project.org).

Target and pathway common analysis. TargetScan Release 7.0 (www.targetscan.org) was employed to predict targets of miR-146a. A total of 2,898 predictions were ranked by the 'cumulative weighted context ++ score', and the top 200 were selected as putative targets of miR-146a for pathway common analysis to identify functional clusters. Pathway enrichment analysis was performed using an online webserver, Webgestalt (www.webgestalt.org) for functional enrichment analysis (11). Pathway analysis was initially used to identify significant linkage of differentially expressed genes. Minimum genes contained in each pathway were set to ' 2 ' and significance ' $\mathrm{P}=0.05$ ' corrected by Benjamini-Hochberg method.

Reverse transcription-quantitative polymerase chain reaction $(R T-q P C R)$. Total RNA was extracted from EBs by TRIzol reagent (Invitrogen; Thermo Fisher Scientific, Inc.), followed by quality assessment. A total of $10 \mathrm{ng}$ total RNA was reverse-transcribed to cDNA using Taqman microRNA Reverse Transcription kit (Applied Biosystems; Thermo Fisher Scientific, Inc.). Quantification of the expression of PDGFRA mRNA was performed using a 7500 Fast Real-Time PCR System. The primers were as follows: PDGFRA forward, 5'-GATCCGGGCTAAGGAAGAAG-3' and reverse, 5'-CCA AAATGGATGCAGGAACT-3'; GAPDH forward, 5'-CAC AATTTCCATCCCAGACC-3' and reverse, 5'-GTGGGT GCAGCGAACTTTAT-3'. GAPDH was used as the internal control. To quantify miR-146a, cDNA was synthesized using mir-XTM miRNA first-strand synthesis and a SYBR qRT-PCR kit (Clontech Laboratories, Inc., Mountainview, CA, USA) according to the manufacturer's protocol. Hsa-miR-146a (forward, 5'-GGGGAGAACTAGGTGCCAAA-3' and reverse, 5'-GCCAGAAAGGAACTTGAACT-3') was used as a primer for RT-qPCR. U6 small nuclear RNA (forward, 5'-GAGAAG GGCTATCCAGGAAG-3' and reverse, 5'-CCGAAAGGA ATTGAAGCACT-3') was used as the internal standard. Initial denaturation was at $94^{\circ} \mathrm{C}$ for $15 \mathrm{~min}$ followed by amplification for 45 cycles $\left(95^{\circ} \mathrm{C}\right.$ for $35 \mathrm{sec}$ and $55^{\circ} \mathrm{C}$ for $\left.50 \mathrm{sec}\right)$. The relative expression was calculated using the $2^{-\Delta \Delta \mathrm{Cq}}$ method (12).

Cell viability assay. Cell viability was detected by methylthiazolyldiphenyl-tetrazolium bromide (MTT) assay (Sigma-Aldrich; Merck KGaA, Darmstadt, Germany). A total of $6 \times 10^{3}$ cells/well were seeded in plates and incubated for $6 \mathrm{~h}$ at $37^{\circ} \mathrm{C}$. Following washing with PBS to remove the non-adherent cells, $10 \mu \mathrm{l}$ MTT reagent was added to each well, and cells were incubated for $3 \mathrm{~h}$ at $37^{\circ} \mathrm{C}$ until purple precipitate was visible. Subsequently, $100 \mu 1$ detergent reagent (Gibco; Thermo Fisher Scientific, Inc.) was added to each well and the plates were kept in the dark for $2 \mathrm{~h}$. The absorbance was measured at a wavelength of $570 \mathrm{~nm}$ on day 1,2 and 3 using an ELx800 Absorbance Reader (BioTek Instruments, Inc., Winooski, VA, USA).

Wound-healing assay. At $24 \mathrm{~h}$ following transfection, $6 \times 10^{3}$ cells were digested with $0.25 \%$ trypsin (Bio-Rad Laboratories, Inc.), centrifuged at $800 \mathrm{x}$ g for $10 \mathrm{~min}$ at $4^{\circ} \mathrm{C}$ and resuspended in RPMI-1640 medium (Gibco; Thermo Fisher Scientific, Inc.) supplemented with $10 \%$ FBS. Cells were seeded as single-cell suspensions of $1 \times 10^{5} / \mathrm{ml}, 1 \mathrm{ml}$ into 6-well plates or as whole-cell monolayers cultured in serum-containing medium (1-2 ml). Adherent cells in the medium were aspirated with a micropipette-like tip to create a $1-\mathrm{mm}$ cell-free zone that was washed with serum-free medium or PBS to remove any remaining cells. Cells were observed under a light microscope $24 \mathrm{~h}$ after transfection and images were captured. 
Transwell assay. At $24 \mathrm{~h}$ following transfection, cells were digested with $0.25 \%$ trypsin, centrifuged at $800 \mathrm{x} \mathrm{g}$ for $10 \mathrm{~min}$ at $4^{\circ} \mathrm{C}$ and resuspended in RPMI-1640 medium supplemented with $10 \%$ FBS. Cells were seeded as single-cell suspensions of $1 \times 10^{5}$ cells/well/200 $\mu \mathrm{l}$ in Transwell chambers with $700 \mu$ RPMI-1640 medium supplemented with $10 \%$ FBS. Cells from the two groups (control and experimental group) were incubated for $48 \mathrm{~h}$ at $37^{\circ} \mathrm{C}$ with $5 \% \mathrm{CO}_{2}$. Cells were fixed in a neutral formalin solution and examined on an inverted microscope at low magnification; three random fields were chosen, and the number of cells was counted under high magnification to determine the mean number of cells per field.

Western blot analysis. Cells $\left(6 \times 10^{3}\right)$ were lysed in NP-40 buffer containing $1 \mathrm{mM}$ phenylmethyl sulfonylfluoride, $150 \mathrm{mM} \mathrm{NaCl}, 1.0 \% \mathrm{NP}-40,50 \mathrm{mM}$ Tris- $\mathrm{HCl}$ and protease inhibitors (Roche Diagnostics, Basel, Switzerland) for $30 \mathrm{~min}$ on ice. Cell lysates were centrifuged at $12,000 \mathrm{x} \mathrm{g}$ for $20 \mathrm{~min}$ at $4^{\circ} \mathrm{C}$ following sonication on ice and supernatants were separated prior to boiling for $10 \mathrm{~min}$ in the presence of 2-mercaptoethanol. Protein concentration was determined using the bicinchoninic assay and $40 \mu \mathrm{g} / \mathrm{lane}$ was separated on $10 \%$ SDS-PAGE and subsequently transferred onto nitrocellulose membranes. Membranes were blocked in $10 \%$ dry milk-TBST [20 mM Tris-HCl (PH 7.5), 0.1\% Tween 20] for $1 \mathrm{~h}$ at $37^{\circ} \mathrm{C}$. Membranes were then washed three times and incubated with monoclonal antibodies against PDGFRA (1:1,000; cat. no. 3164; Cell Signaling Technology, Inc., Danvers, MA, USA) and GAPDH (1:1,000; cat. no. 2118; Cell Signaling Technology, Inc.) overnight at $4^{\circ} \mathrm{C}$. The resultant membranes were washed and incubated with a horseradish peroxidase-conjugated goat anti-rabbit secondary antibody (1:3,000; cat. no. sc-2004; Santa Cruz Biotechnology, Inc.) in TBST at $4^{\circ} \mathrm{C}$ overnight. Bands were visualized with an enhanced chemiluminescence kit (GE Healthcare, Chicago, IL, USA).

Luciferase reporter gene assay. The 3'-UTR of PDGFRA was amplified by PCR and inserted into the pGL3-controlvector (Promega Corporation, Madison, WI, USA). To generate the PDGFRA3'-UTR mutant reporter, the seed region of PDGFRA 3'-UTR was mutated to remove all complementarity to nucleotides 17-22 of miR-146a, using the QuikChangeII XL mutagenesis kit (Stratagene; Agilent technologies, Inc., Santa Clara, CA, USA). The PDGFRA3'-UTR mutant was further verified by sequencing. Cells were seeded in a 24 -well plate at a density of $1 \times 10^{5}$ cells/well. Following a 24-h culture, cells were co-transfected with miR-146a and firefly luciferase reporter plasmids containing WT or PDGFRA 3'-UTR mutant, using Lipofectamine 2000 (Invitrogen; Thermo Fisher Scientific, Inc.). At $36 \mathrm{~h}$ following transfection, firefly and Renilla luciferase activities were measured by Dual Luciferase Reporter assay (Promega Corporation). The assay was performed in triplicate.

Spheroid sprouting assay. Multicellular spheroids were generated by seeding $1 \times 10^{4}$ cells/well in 96-well plates filled with DMEM (Gibco; Thermo Fisher Scientific, Inc.) containing 5\% FBS and $0.24 \%$ high-viscosity methylcellulose (Tecan Group, Ltd., Männedorf, Switzerland). Spheroids were collected following culture at $37^{\circ} \mathrm{C}$ for $24 \mathrm{~h}$, and embedded in collagen gels and kept in DMEM containing $2 \% \mathrm{FBS}$ at $37^{\circ} \mathrm{C}$ for another $24 \mathrm{~h}$. AOBs were prepared from the leaves of Phyllostachys nigra var. henonis (Zhejiang University Innoessen Bio-Technology Co., Ltd). AOB was added at a final concentration of $100 \mu \mathrm{g} / \mathrm{ml}$ from a stock solution of $10 \mathrm{mg} / \mathrm{ml}$. Following treatment for $24 \mathrm{~h}$ at $4^{\circ} \mathrm{C}$, cells were observed under a Zeiss Axiovert 25 microscope (Carl Zeiss AG, Oberkochen, Germany) and analyzed using the KS400 Kontron image analysis software (version 1.2, Kontron Elektronik GmbH, Munich, Germany).

Statistical analysis. GraphPad Prism 5 software (version 5; GraphPad Software, Inc., La Jolla, CA, USA) was used to perform the statistical analysis. Data is presented as the mean \pm standard error and was analyzed using the one-way analysis of variance. Multiple comparisons between the groups were performed using the Student-Newman-Keuls method. $\mathrm{P}<0.05$ was considered to indicate a statistically significant difference.

\section{Results}

$A O B$ modulates microRNA expression in EBs. In this study, treatment with $\mathrm{AOB}$ increased the proliferation and migration of EBs (Fig. 1A and B, respectively). To further detect the microRNA expression profile involved in AOB-treated EBs, microarray analysis was conducted. As depicted in Fig. 1, expression differences were compared between AOB-treated and the control cells. In all, 15 miRNAs were downregulated, among which miR-146a was the most downregulated one, followed by miR-376. Significant differences were also identified in miRNA-294, miRNA-224 and miR-373 (Fig. 1C). RT-qPCR results demonstrated that the expression levels of miR-146a (log $\mathrm{FC}=-1.97, \mathrm{P}=2.03 \mathrm{E}-11)$ and $\mathrm{miR}-376(\log \mathrm{FC}=-1.23, \mathrm{P}=6.49 \mathrm{E}-13)$ were both markedly downregulated following AOB treatment compared with the control (Fig. 1D).

miR-146a regulates $E B$ proliferation. To examine whether miR-146a is essential in the angiogenesis process following AOB treatment, miR-146a antagomir or mimic was transfected into EBs, and the proliferation was determined. As illustrated in Fig. 2A, transfection of miR-146a antagomir significantly reduced the expression of miR-146a, whereas transfection of miR-146a mimic markedly increased the level of miR-146a, compared with transfection with the scramble. The cell viability of EBs was continually measured for 3 days following transfection of miR-146a antagomir or mimic. The results demonstrated that the growth rate of EBs transfected with miR-146a antagomir was higher than that of EBs transfected with the scramble sequence. By contrast, cells transfected with miR-146a mimics had an increased growth rate compared with the control cells transfected with the scramble sequence (Fig. 2B).

Putative targets of miR-146a are abundant in the phosphatidylinositol 3-kinase/protein kinase $B(P I 3 K / A K T)$ signaling pathway. By Targetscan software, a total of 1,327 transcripts were predicted as putative targets of miR-146a, namely the 'cumulative weighted context ++ score'. Genes were chosen for the pathway common analysis. These predicted genes were enriched in various pathways, including 
A

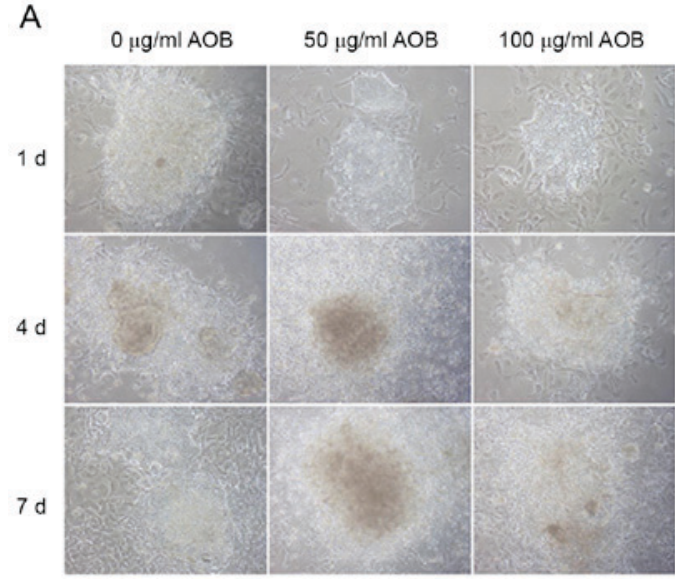

C

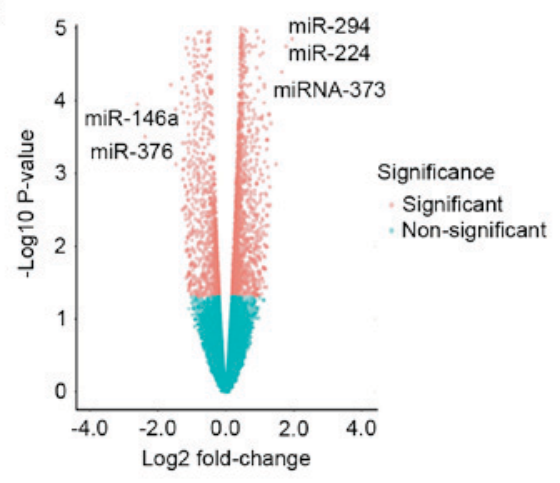

B
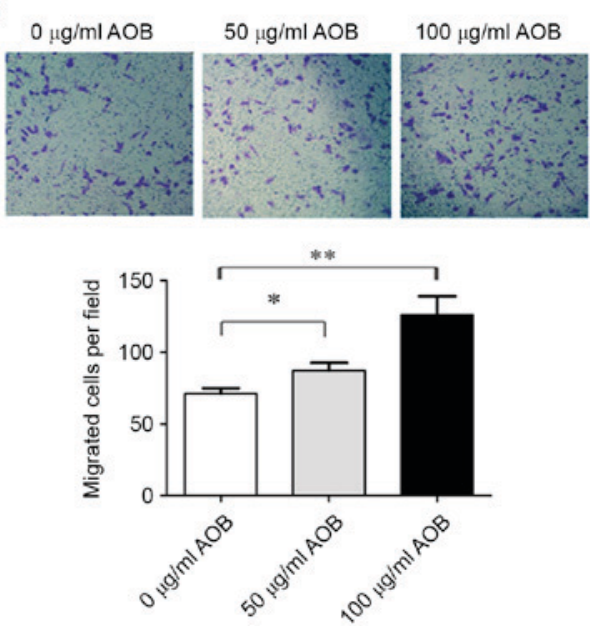

D

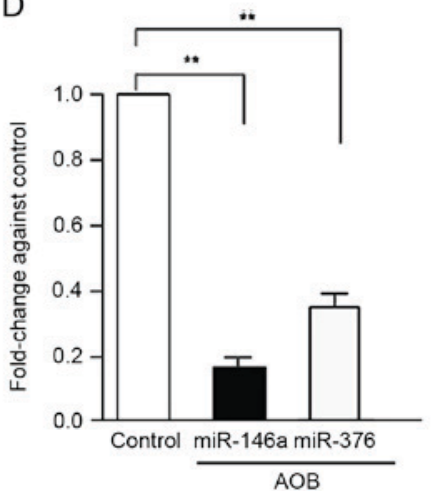

Figure 1. AOB treatment of EB and the differentially expressed miRNAs between AOB-treated and control cells. AOB treatment promotes the (A) proliferation (magnification, $\mathrm{x} 400$ ) and (B) the migration of EBs (magnification, x100). (C) Volcano plot of the miRNA profile in EBs treated with AOB. Red dots represent significantly expressed miRNAs, and green dots represent the non-significantly expressed ones. (D) Cells were treated with or without (control) $\mathrm{AOB}$, and miR-146a and miR-376 mRNA levels were determined by reverse transcription-quantitative polymerase chain reaction. Data are expressed as the mean \pm standard error. ${ }^{* *} \mathrm{P}<0.01$ vs. the control. $\mathrm{EB}$, embryoid body; $\mathrm{AOB}$, antioxidant of bamboo leaves; miR, microRNA.

A

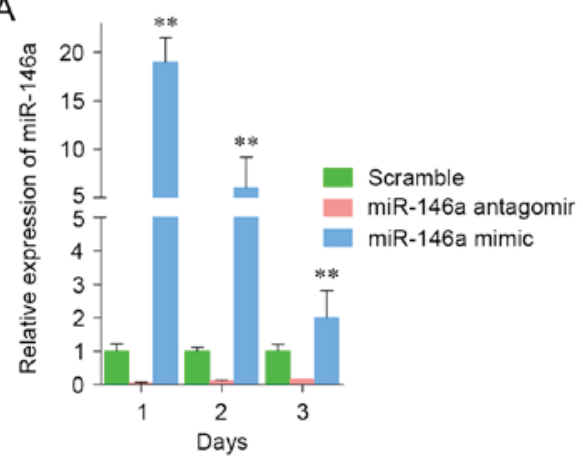

B

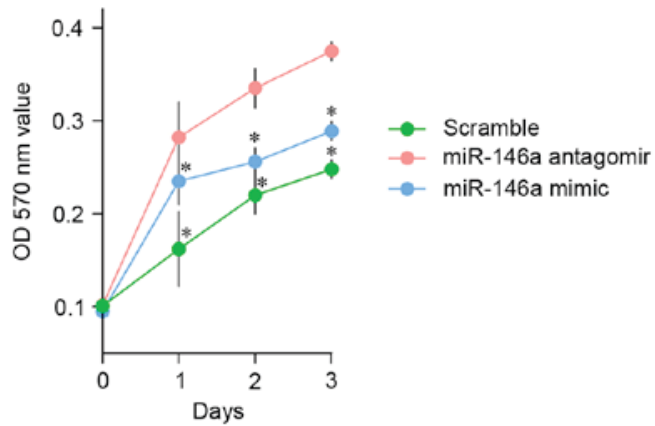

Figure 2. miR-146a mRNA expression and cell viability of EBs following transfection with miR-146a antagomir or mimic. (A) mRNA expression levels of miR-146a was measured by reverse transcription-quantitative polymerase chain reaction. Error bars indicate standard errors. Statistical significance was determined by one-way analysis of variance. (B) Cell viability of EBs following transfection with miR-146a antagomir, mimic or scramble sequences as assessed using the MTT method. Data is expressed as the mean \pm standard error. ${ }^{*} \mathrm{P}<0.05,{ }^{* *} \mathrm{P}<0.01$ vs. scramble. AOB, antioxidant of bamboo leaves; EB, embryonic body; miR, microRNA; OD, optical density.

the 'wingless-type MMTV integration site (Wnt) signaling pathway', the 'vascular endothelial growth factor (VEGF) and VEGF receptor (VEGFR) signaling network', the 'PI3K/AKT pathway', 'Endothelins', and the 'mitogen-activated protein kinase (MAPK) signaling network' (Table I). In particular, the 'PI3K/AKT pathway' and the 'VEGF/VEGFR signaling network pathway' were involved in angiogenesis, indicating that miR-146a might regulate angiogenesis through them.

PDGFRA is a target of miR-146a. PDGFRA has been reported to be associated with angiogenesis and cell migration (13). Therefore, it was postulated that miR-146a might function via targeting PDGFRA. To access this assumption, the mRNA and 
Table I. Over represented pathways of top 200 predicted target genes of microRNA-146a.

\begin{tabular}{|c|c|c|c|c|}
\hline ID & Pathway & Observed & Expected & $\operatorname{adj} P$ \\
\hline 1585 & Arf6 downstream pathway & 22 & 5.65 & $3.2 \times 10^{7}$ \\
\hline 1575 & VEGF and VEGFR signaling network & 22 & 5.64 & $3.3 \times 10^{7}$ \\
\hline 1556 & Plasma membrane estrogen receptor signaling & 22 & 5.71 & $3.5 \times 10^{5}$ \\
\hline 1472 & Nectin adhesion pathway & 22 & 5.73 & $3.7 \times 10^{5}$ \\
\hline 1461 & GMCSF-mediated signaling events & 22 & 5.65 & $3.7 \times 10^{7}$ \\
\hline 1615 & Arf6 trafficking events & 22 & 5.65 & $3.7 \times 10^{7}$ \\
\hline 15744 & Signaling events mediated by focal adhesion kinase & 22 & 5.64 & $3.4 \times 10^{7}$ \\
\hline 1602 & ErbB1 downstream signaling & 22 & 5.64 & $3.4 \times 10^{5}$ \\
\hline 1571 & mTOR signaling pathway & 22 & 5.64 & $3.4 \times 10^{5}$ \\
\hline 1619 & Endothelins & 22 & 5.71 & $3.4 \times 10^{7}$ \\
\hline 1517 & Beta1 integrin cell surface interactions & 22 & 5.90 & $5.6 \times 10^{7}$ \\
\hline 1499 & Integrin family cell surface interactions & 22 & 6.01 & $7.66 \times 10^{7}$ \\
\hline 1546 & Integrin-linked kinase signaling & 13 & 2.19 & $2.63 \times 10^{5}$ \\
\hline 1488 & CDC42 signaling events & 13 & 3.36 & 0.0001 \\
\hline 1544 & E-cadherin signaling in the nascent adherens junction & 7 & 1.23 & 0.0006 \\
\hline 1494 & $\mathrm{~N}$-cadherin signaling events & 6 & 1.12 & 0.0023 \\
\hline 1611 & Regulation of nuclear SMAD2/3 signaling & 6 & 1.35 & 0.0055 \\
\hline 936 & Cell junction organization & 3 & 0.02 & 0.0074 \\
\hline 935 & Cell-Cell communication & 3 & 0.58 & 0.0245 \\
\hline
\end{tabular}

'adjP' represents Benjamini-Hochberg corrected P-value; 'Observed' denotes the number of overlapped genes between queried and pathway gene set, and 'Expected' stands for the expected number of randomly sampled genes with the same size with the queried set in this pathway.

A

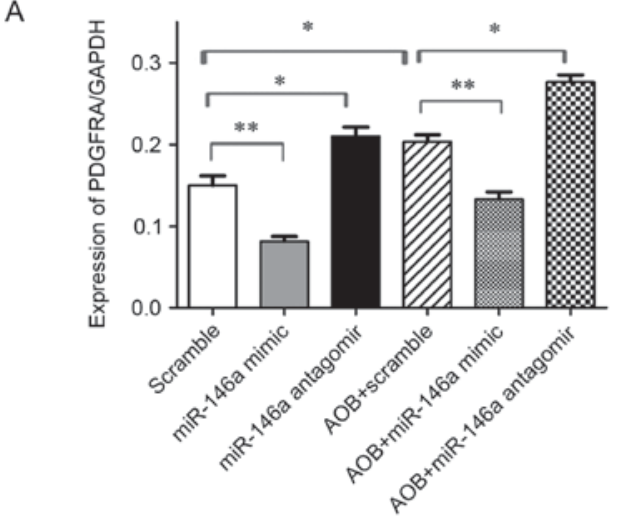

C WT-PDGFRA 3'-UTR 5' AAAAgUuACAUGUUUGCACCUUU miR-146a 3' GAUUGACGUGAUCUACGUGGAAU MT-PDGFRA 3'-UTR 5' AAAAGUUACAUGUUUAUCGAGCU
B
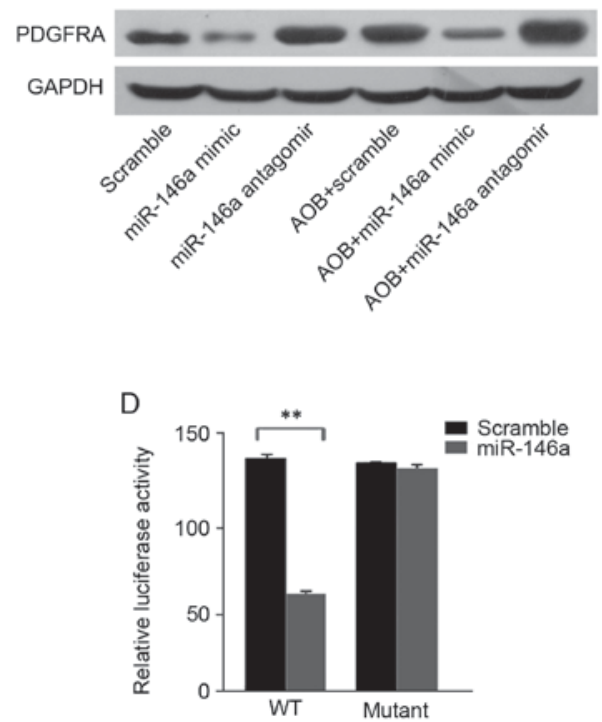

Figure 3. PDGFRA mRNA and protein expression in EBs transfected with miR-146a mimic or antagomir following AOB treatment. EBs were transfected with miR-146a mimic, antagomir, or scramble sequence following treatment without or with $0.6 \mathrm{mg} / \mathrm{ml}$ AOB and PDGFRA expression was determined at (A) mRNA and (B) protein levels. 'P<0.05; ${ }^{* *} \mathrm{P}<0.01$. (C) Sequences of PDGFRA 3'-UTR (WT-PDGFRA 3'-UTR) and PDGFRA 3'-UTR mutant (MT-PDGFRA 3'-UTR) for miR-146a binding. (D) Relative luciferase activity of miR-146a mimic + WT vs. miR-146a mimic + Mutant. Data are expressed as the mean \pm standard error from three independent experiments $(n=3) .{ }^{* *} \mathrm{P}<0.01$ vs. scramble. PDGFRA, platelet-derived growth factor receptor alpha; AOB, antioxidant of bamboo leaves; miR, microRNA; UTR, untranslated region; WT, wild type; EB, embryonic body.

protein expression levels of PDGFRA in EBs was determined following transfection with miR-146a mimic or antagomir, using RT-qPCR and western blotting, respectively. The results demonstrated that transfection with miR-146a mimics significantly decreased both mRNA and protein expression levels of
PDGFRA, whereas transfection with miR-146a antagomir led to a remarkable increase of PDGFRA expression. In addition, AOB treatment markedly elevated the level of PDGFRA (Fig. 3A and B). Notably, transfection with miR-146a mimic obstructed AOB-mediation PDGFRA upregulation (Fig. 3A and B), while 
A

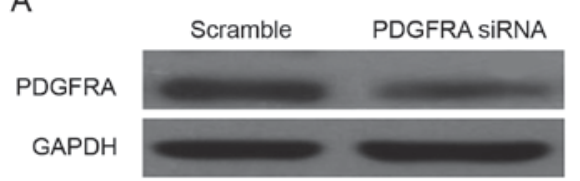

B

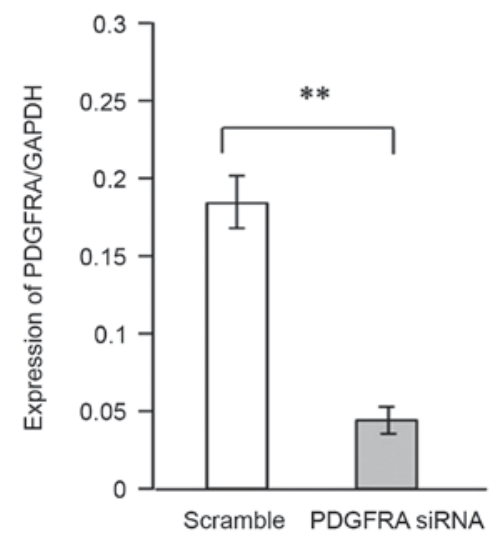

Figure 4. Knockdown efficiency of PDGFRA siRNA. Assessed by (A) western blotting and (B) by reverse transcription-quantitative polymerase chain reaction. Error bars indicate standard errors. Data are expressed as the mean \pm standard error. ${ }^{* *} \mathrm{P}<0.01$ vs. scramble. PDGFRA, platelet-derived growth factor receptor alpha; AOB, antioxidant of bamboo leaves; siRNA, small interfering RNA.

A

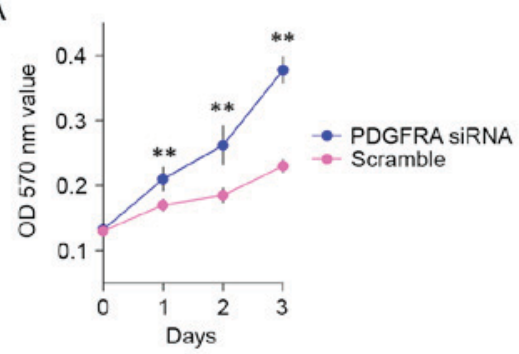

B

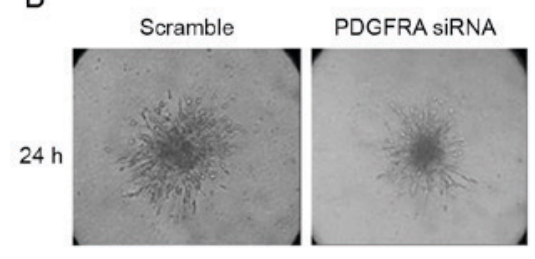

C

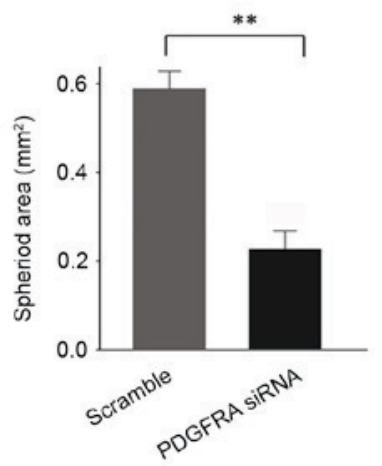

Figure 5. Studying (A) cell viability as assessed by MTT and (B) spheroid sprouting assay in EBs transfected with PDGFRA or scramble siRNA at $24 \mathrm{~h}$. Magnification x20. (C) Quantification of vessel sprouting of EBs transfected with PDGFRA siRNA as assessed by the angiogenesis assay. Data are expressed as the mean \pm standard error. ${ }^{* *} \mathrm{P}<0.01$ vs. scramble. PDGFRA, platelet-derived growth factor receptor alpha; $\mathrm{AOB}$, antioxidant of bamboo leaves; EB, embryonic body; siRNA, small interfering RNA.
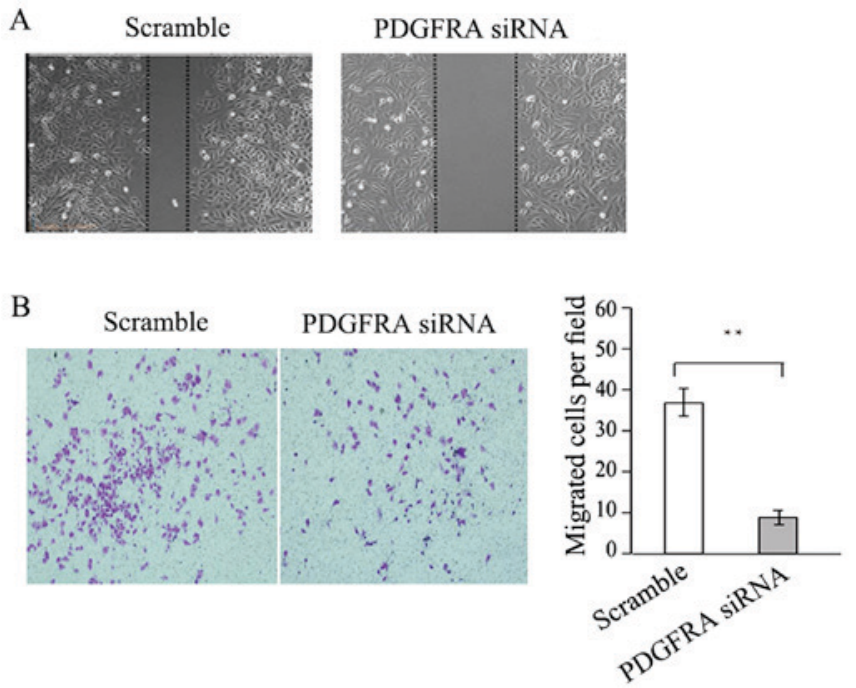

Figure 6. Migration of EBs following PDGFRA knockdown, assessed by (A) wound-healing and (B) Transwell assay in EBs transfected with PDGFRA or scramble siRNA. Data are expressed as the mean \pm standard error. ${ }^{* *} \mathrm{P}<0.01$. PDGFRA, platelet-derived growth factor receptor alpha; AOB, antioxidant of bamboo leaves; EB, embryonic body; siRNA, small interfering RNA.

transfection of miR-146a antagomir led to a further increase in PDGFRA expression following AOB treatment (Fig. 3A and B). To further confirm that PDGFRA is a target of miR-146a, a dual luciferase reporter assay was performed (Fig. 3C). PDGFRA 3'-UTR or PDGFRA 3'-UTR mutant was co-transfected with miR-146a mimics into EBs, and $36 \mathrm{~h}$ following this, the luciferase activity was detected. The result illustrated that miR-146a mimic decreased the luciferase activity of PDGFRA 3'-UTR but had a minimal effect on that of PDGFRA 3'-UTR mutant (Fig. 3D), suggesting that PDGFRA is a target of miR-146a.

Silencing of PDGFRA inhibits the proliferation and migration induced by $A O B$. Whether PDGFRA is involved in AOB-mediated proliferation and migration of EBs was also investigated. The cell viability and angiogenic sprouting of EBs were measured following PDGFRA knockdown and AOB treatment. Knockdown efficiency of PDGFRA was confirmed at the protein and mRNA levels (Fig. 4A and B, respectively). Cell viability analysis demonstrated that silencing of PDGFRA significantly increased the growth rate of EBs (Fig. 5A). PDGFRA-ablated EBs demonstrated decreased angiogenic sprouting in comparison with the control cells (Fig. 5B and C). In addition, PDGFRA knockdown significantly reduced the migration ability of EBs as demonstrated by the wound-healing assay and Transwell assay (Fig. 6A and B, respectively). Together, PDGFRA deficiency inhibited EB proliferation and migration, demonstrating a vital role in EB angiogenesis.

\section{Discussion}

ESCs can be induced under certain conditions to form ectoderm, mesoderm and endoderm cell types, including EBs (14). EBs are basically formed during early embryonic development of embryos following gastrulation, and are easy to handle and to carry out various operations, avoiding 
experiments with embryos and ethical controversy $(15,16)$. Therefore, EB is a preferred experimental model for studying early embryonic development, genetic, mesoderm mutual induction, the impact of environmental factors on the development of the early embryos, exogenous substances embryonic teratogenic, cytotoxic and tire-borne diseases and other diseases (17). In recent years, increasing research on miRNA has emphasized the importance of miRNAs in gene regulation (18). miRNAs are a class of non-coding single-stranded small RNA nucleotides from pri-miRNA processing by Drosha and Dicer (19). Typically, miRNAs can recognize specific mRNAs, and bind to their 3'-UTR by inexact match, affecting mRNA stability, or inhibit their translation, regulating the expression levels of target genes (20-22). Studies on miRNA functions are mainly achieved with the usage of in vitro miRNA mimics.

The Wnt signaling pathway is recognized as the most associative pathway to epithelialization. In the Wnt signaling pathway, PDGFRA which has been reported to function in angiogenesis, was further verified as a target of miR-146a $(23,24)$. Zhu et al $(25)$ have reported that miR-146a can target PDGFRA 3'-UTR, and its aberrant expression markedly decreases PDGFRA expression, which is associated with the activity of EC angiogenesis in hepatic cell carcinoma. This report indicated that the processes of angiogenesis and cancer metastasis to some extent share common biological signaling pathways. Based on the above statements, PDGFRA is identified as a vital element in angiogenesis.

In conclusion, changes in the miRNA profile in AOB-treated EBs were explored, among which miR-146a was demonstrated to be the most susceptible one. Although target prediction yielded a large number of candidates, the list was narrowed by functional enrichment analysis. Among all putative pathways, five were highly implicated in angiogenesis, suggesting a target pool of miR-146a, and PDGFRA was verified as one of its targets. Although the underlying mechanism remains to be elucidated, the present study implies the potential therapeutic value of AOB on EB angiogenesis via downregulation of miR-146a. Furthermore, the functional clusters identified based on the putative targets of miR-146a may offer candidates for further exploring their role in angiogenesis and associated diseases.

\section{References}

1. Ma X, Wang E, Lu Y, Wang Y, Ou S and Yan R: Acylation of antioxidant of bamboo leaves with fatty acids by lipase and the acylated derivatives' efficiency in the inhibition of acrylamide formation in fried potato crisps. PLoS One 10: e0130680, 2015.

2. Liu L, Xia B, Jin C, Zhang Y and Zhang Y: Chemical acylation of water-soluble antioxidant of bamboo leaves (AOB-w) and functional evaluation of oil-soluble AOB (cAOB-o). J Food Sci 79: C1886-C1894, 2014.

3. Zhang Y, Luo Z, Shao Z, Yu C and Wang S: Effects of antioxidants of bamboo leaves and flavonoids on 2-amino-1-methy 1-6-phenylimidazo[4,5-b]pyridine (PhIP) formation in chemical model systems. J Agric Food Chem 62: 4798-4802, 2014.

4. Hirota S and Ogawa M: Activin A in combination with OP9 cells facilitates development of Flk-1(+) PDGFR $\alpha(-)$ and Flk-1(+) PDGFR $\alpha(+)$ hematopoietic mesodermal cells from murine embryonic stem cells. Biochem Biophys Res Commun 467: 583-588, 2015

5. Moriya $\mathbf{J}$ and Ferrara N: Inhibition of protein kinase $\mathrm{C}$ enhances angiogenesis induced by platelet-derived growth factor $\mathrm{C}$ in hyperglycemic endothelial cells. Cardiovasc Diabetol 14: 19, 2015.
6. Ro S, Park C, Young D, Sanders KM and Yan W: Tissue-dependent paired expression of miRNAs. Nucleic Acids Res 35: 5944-5953, 2007.

7. Mallory AC and Vaucheret H: MicroRNAs: Something important between the genes. Curr Opin Plant Biol 7: 120-125, 2004.

8. Hayes J, Peruzzi PP and Lawler S: MicroRNAs in cancer: Biomarkers, functions and therapy. Trends Mol Med 20: 460-469, 2014.

9. Chang TY, Huang TS, Wang HW, Chang SJ, Lo HH, Chiu YL, Wang YL, Hsiao CD, Tsai CH, Chan CH, et al: miRNome traits analysis on endothelial lineage cells discloses biomarker potential circulating microRNAs which affect progenitor activities. BMC Genomics 15: 802, 2014.

10. Sundaravinayagam D, Kim HR, Wu T, Kim HH, Lee HS, Jun S, Cha JH, Kee Y, You HJ and Lee JH: miR146a-mediated targeting of FANCM during inflammation compromises genome integrity. Oncotarget 7: 45976-45994, 2016.

11. Zhang B, Kirov S and Snoddy J: WebGestalt: An integrated system for exploring gene sets in various biological contexts. Nucleic Acids Res 33 (Web Server Issue): W741-W748, 2005.

12. Livak KJ and Scmittgen TD: Analysis of relative gene expression data using real-time quantitative PCR and the 2(-Delta Delta C(T)) method. Methods 25: 402-408, 2001.

13. McCarthy N, Liu JS, Richarte AM, Eskiocak B, Lovely CB, Tallquist MD and Eberhart JK: Pdgfra and Pdgfrb genetically interact during craniofacial development. Dev Dyn 245: 641-652, 2016.

14. Dziedzicka D, Markouli C, Barbé L, Spits C, Sermon K and Geens M: A high proliferation rate is critical for reproducible and standardized embryoid body formation from laminin-521-based human pluripotent stem cell cultures. Stem Cell Rev 12: 721-730, 2016.

15. Boxman J, Sagy N, Achanta S, Vadigepalli R and Nachman I: Integrated live imaging and molecular profiling of embryoid bodies reveals a synchronized progression of early differentiation. Sci Rep 6: 31623, 2016.

16. Moon SH, Ju J, Park SJ, Bae D, Chung HM and Lee SH: Optimizing human embryonic stem cells differentiation efficiency by screening size-tunable homogenous embryoid bodies. Biomaterials 35: 5987-5997, 2014.

17. Kim JE, Lee JM and Chung BG: Microwell arrays for uniform-sized embryoid body-mediated endothelial cell differentiation. Biomed Microdevices 16: 559-566, 2014.

18. Crabbé MA, Gijbels K, Visser A, Craeye D, Walbers S, Pinxteren J, Deans RJ, Annaert W and Vaes BL: Using miRNA-mRNA interaction analysis to link biologically relevant miRNAs to stem cell identity testing for next-generation culturing development. Stem Cells Transl Med 5: 709-722, 2016

19. Yang Y, Xing Y, Liang C, Hu L, Xu F and Chen Y: Crucial microRNAs and genes of human primary breast cancer explored by microRNA-mRNA integrated analysis. Tumour Biol 36: 5571-5579, 2015.

20. Tutar L, Tutar E, Özgür A and Tutar Y: Therapeutic targeting of microRNAs in cancer: Future perspectives. Drug Dev Res 76: 382-388, 2015.

21. Breda J, Rzepiela AJ, Gumienny R, van Nimwegen E and Zavolan M: Quantifying the strength of miRNA-target interactions. Methods 85: 90-99, 2015.

22. Miao CG, Xiong YY, Yu H, Zhang XL, Qin MS, Song TW and Du CL: Critical roles of microRNAs in the pathogenesis of systemic sclerosis: New advances, challenges and potential directions. Int Immunopharmacol 28: 626-633, 2015.

23. Rudat C, Norden J, Taketo MM and Kispert A: Epicardial function of canonical Wnt-, Hedgehog-, Fgfr1/2- and Pdgfra-signalling. Cardiovasc Res 100: 411-421, 2013.

24. Li F, He Z, Li Y, Liu P, Chen F, Wang M, Zhu H, Ding X, Wangensteen $\mathrm{KJ}, \mathrm{Hu} \mathrm{Y}$ and Wang X: Combined activin $\mathrm{A} / \mathrm{LiCl} / \mathrm{Noggin}$ treatment improves production of mouse embryonic stem cell-derived definitive endoderm cells. J Cell Biochem 112: 1022-1034, 2011.

25. Zhu K, Pan Q, Zhang X, Kong LQ, Fan J, Dai Z, Wang L, Yang XR, Hu J, Wan JL, et al: MiR-146a enhances angiogenic activity of endothelial cells in hepatocellular carcinoma by promoting PDGFRA expression. Carcinogenesis 34: 2071-2079, 2013. 